\title{
EL FORMADOR DE FUTUROS PROFESIONALES: UNA NUEVA FORMA DE COMPRENDER LA DOCENCIA EN LA EDUCACIÓN SUPERIOR UNIVERSITARIA
}

\section{Antecedentes de tener en cuenta en esta discusión}

A partir de la década de los ochenta un tema que ha comenzado a discutirse ampliamente en el mundo son los procesos de enseñanza y aprendizaje que se dan en la educación superior, dado que el aumento de la cobertura y complejidad en la educación terciaria ha generado como consecuencia nuevos desafíos para los docentes universitarios (Ashwin, 2006; Biggs, 2003; Brunner, 2008; D’Andrea y Gosling, 2005; Knight, 2005; OCDE, 2009).

Prueba de esto es que se observa una extensa literatura, tanto teórica como empírica, relacionada con este tema (Kreber, 2002b; Martin et al., 2000; Prosser y Trigwell, 1999; Ramsden, 2003). Lo interesante de la discusión es que se comienza a debatir y visibilizar al interior de las universidades la sobrevaloración de la producción de nuevos conocimientos desarrollados a través de una investigación disciplinar en contraposición a otras labores académicas como la integración, aplicación y transmisión de estos (Boyer, 1990; Brew, 2003; Kreber, 2002a).

En este sentido, tradicionalmente en las comunidades académicas se otorga mayor valor a las tareas de investigación por sobre las de docencia, vinculándose, en la mayoría de los casos, el desarrollo académico de los docentes universitarios más a su productividad científica como investigador que a sus competencias o destrezas como educador (Harland y Staniforth, 2008). Sin embargo, los académicos, además de investigar, la mayoría de las veces enseñan y forman a futuros profesionales, lo cual implica un conjunto de destrezas específicas distintas a las de ser investigador, las que debiesen ser tomadas en cuenta. 
Los docentes que participan en la formación de futuros profesionales no sólo transmiten contenidos respecto de una disciplina sino también un ethos profesional asociado a ésta (Becher, 2001). Asimismo, la mayoría de las veces los docentes son profesionales que saben de su disciplina, pero que no necesariamente aprendieron cómo enseñarla (para ejemplificar, los docentes que educan a futuros ingenieros, en su etapa de formación se focalizaron en aprender las competencias asociadas a la disciplina y no necesariamente en cómo en enseñar ingeniería). Del mismo modo, los aportes de Shulman (1987, 1999) en el estudio del conocimiento profesional de la enseñanza señalan que ella se sustenta en el dominio de un conjunto de conocimientos (pedagógicos, curriculares, de los estudiantes, del contexto en donde se instruye y contenido que se enseña, entre otros), por lo que no basta con saber un contenido específico para ser capaz de enseñarlo.

La ausencia de políticas y prácticas enfocadas en la formación profesional ha tenido un impacto en las aulas de acuerdo a lo que se indica en el informe OCDE (2009). Éste señala que al interior de las salas de clases universitarias prevalecen aún métodos educativos tradicionales en los cuales se privilegia la memorización de los contenidos, el aprendizaje individual y la reproducción en la evaluación de los aprendizajes desarrollados por los estudiantes.

Asimismo, Brunner (2008, p. 128) sostiene que esta visión tradicional de la enseñanza universitaria se caracteriza por ser eminentemente profesionalizante, en donde se privilegian los métodos de enseñanza centrados en los contenidos y los estudiantes poseen un rol pasivo, por lo que "el aprendizaje continúa siendo definido como un proceso de adquisición de conocimientos, débilmente entrelazado con la práctica reflexiva que supone forma el núcleo de las profesiones".

Lo anteriormente expuesto cobra relevancia si se considera que los docentes universitarios son las personas encargadas de la formación de futuros profesionales. Son ellos quienes definen las prácticas educativas que se implementan al interior de la sala de clase, así como las transformaciones metodológicas y didácticas del 
currículo que se deben realizar para enfrentar las nuevas demandas del entorno laboral (Imbernon, 2000; Navio, 2006).

Desde esta perspectiva, se hace imperativo comenzar a profundizar en el papel que los docentes cumplen al interior de la sala de clase visualizándolo no sólo como un profesional experto de una disciplina, sino también como uno que posee una experticia o habilidad específica para poder enseñarla.

De la misma forma, si queremos mejorar los procesos de enseñanza y aprendizaje que se implementan al interior de las aulas universitarias debemos ser críticos y reflexionar no solo sobre lo que se enseña y cómo sino que también quién enseña. Esto implica resituar la mirada en los docentes universitarios, aportándole nuevos significados a la tarea que realizan y considerando la complejidad de los procesos de enseñanza y aprendizaje propios de estos contextos educativos.

Dado lo anteriormente expuesto, este artículo propone una nueva aproximación a esta problemática considerando ciertos aspectos relacionados con la tarea de formar a futuros profesionales que han sido poco debatidos y analizados al interior de la educación superior, los cuales se profundizarán a continuación.

\section{El formador universitario: una nueva forma de comprender al docente universitario}

Para una mejor comprensión, se abordan dos temas que se consideran claves de tener en cuenta en esta discusión: en primer lugar, la visualización de los profesores universitarios como profesionales de un área disciplinar que se desempeñan como educadores formando a otras personas; y, en segundo lugar, la modalidad en que estos profesionales aprenden a enseñar su disciplina en los distintos contextos de formación profesional.

\section{1) ¿Qué entendemos por formador universitario?}

Tradicionalmente al interior de las universidades, los docentes son profesionales de distintas áreas disciplinares que enseñan sus 
conocimientos a futuros profesionales. En la mayoría de los casos no han sido preparados formalmente en la disciplina de enseñar, por lo que la experticia de enseñar la han desarrollado a través de una formación continua más que inicial (Imbernon, 1999; Navio, 2006). Esto cobra relevancia si se toma en cuenta que en Chile, así como en otros países latinoamericanos y anglosajones, para hacer clases en instituciones de educación superior no es necesario tener un título o habilitación profesional de educador.

Se considera fundamental comenzar a concebir a los docentes universitarios más como "formadores" que como "profesores" universitarios. Esta distinción se sustenta en que el término "formador" se entiende como

“...aquel profesional relacionado con la formación en y para el trabajo y que, por tanto, desarrolla tareas tan variadas como la planificación, el diseño, el desarrollo y/o la evaluación curricular, así como la investigación y la innovación en su quehacer diario en contextos globales y locales que condicionan su actuación y en los que interviene activamente" (Navio, 2006, p. 64).

Desde esta perspectiva, los formadores son aquellas personas que de alguna manera tienen relación con la educación de otros individuos, ya sea en la planificación o realización de la misma. A su vez, poseen la habilidad de poder mediar los conocimientos que dominan a personas que desean adquirirlos, desarrollando dicha experticia por medio de un conocimiento práctico.

Comenzar a conceptualizar a los docentes universitarios como formadores universitarios cobra sentido si considera que esta definición permitiría responder y delimitar de manera más apropiada al conjunto de profesionales que, en diferentes contextos y desde distintos referentes profesionales y personales, actúan en el amplio campo de la formación universitaria (Navio, 2006). De este modo, se connota que los docentes universitarios, más que profesores son profesionales que se preocupan de formar a otras personas y que, en dicha tarea, precisan de un conjunto de destrezas específicas relacionadas con la docencia. 
$\mathrm{Al}$ respecto, en décadas pasadas se esperaba que los académicos que enseñaban en las universidades sólo tenían que ser expertos en sus materias ya que el aprendizaje de los estudiantes se consideraba como un tema no problemático o de poca relevancia (Ashwin, 2006). Sin embargo, actualmente se espera que los académicos y otros profesionales que apoyan en la formación profesional estén preparados para desempeñar su papel como educadores, tanto en los procesos de enseñanza-aprendizaje así como en el contenido que enseñan.

Esta nueva demanda en el ejercicio profesional como formador universitario se puede apreciar en las políticas y en la Ley de Aseguramiento de la Calidad de la Educación Superior, institucionalizada por la Comisión Nacional de Acreditación (CNA), en el año 2006. Uno de los aspectos que se supervisan en los procesos de acreditación institucional y de carreras es la docencia que se imparte, en donde se evalúan los métodos pedagógicos y estrategias de enseñanza y aprendizaje así como los resultados obtenidos en el uso de dichas metodologías con el propósito de asegurar la calidad de los aprendizajes en estos espacios educativos (CNA, 2010).

Este requerimiento por fortalecer la calidad del aprendizaje en la formación profesional se sustenta en la necesidad de desarrollar profesionales preparados para enfrentar la complejidad e incertidumbre del entorno laboral en el cual se insertarán, para lo cual precisarán de diversas competencias tales como: capacidad de trabajar colaborativamente, de gestionar el conocimiento, de indagación para la resolución de problemas y de aprendizaje continuo, entre otras (Barnett, 2000).

Lo expresado anteriormente presupone la presencia de formadores con un amplio dominio no sólo disciplinar sino también pedagógico y reflexivo de lo que enseña, visualizando la complejidad de esta tarea. Por ende, y tal como lo señala Shulman (2000), los profesionales que se desempeñan como formadores universitarios debiesen concebirse como personas que poseen al menos dos profesiones: la propia (del campo profesional o disciplinar de origen) y la de educador. 
Sin embargo, esta visión más integral del formador universitario se percibe con dificultad al interior de la educación superior universitaria, lo cual se puede comprender por dos motivos: por una parte, la tendencia de sustentar y legitimar el desarrollo profesional continuo de los académicos en el dominio de su área disciplinar y destreza como investigador, siendo la tarea de enseñar relegada a una función de segunda prioridad; y, por otra, que los académicos con dificultad se identifican con la palabra "educador", rechazando este papel, incluso en ocasiones en su totalidad, e identificándose con mayor cercanía a la identidad profesional propia de su formación disciplinar de origen (Ashwin, 2006; D’Andrea y Gosling, 2005).

\section{2) ¿Cómo aprenden a enseñar los formadores universitarios?}

Otro tópico interesante de profundizar tiene relación con la experticia para poder enseñar que desarrollan los formadores universitarios a lo largo de su trayectoria profesional como educadores. La importancia de este tema radica en visualizar la docencia de los formadores universitarios como una actividad provista teóricamente y no como un simple proceso de transmisión de nuevos conocimientos e información (Shulman, 1999; Trigwell, 2001).

Esta premisa plantea la necesidad de discutir la tendencia de concebir la enseñanza como un "oficio" que puede ser aprendido de manera familiar sin un tipo de esfuerzo intelectual o teórico (Comisión Boyer 1998, citado en Ashwin, 2006). Una forma de dimensionar este tema se logra gracias al aporte que Navio (2006) propone respecto de las principales orientaciones en la formación docente, las cuales se pueden apreciar en la siguiente tabla: 
Tabla 1. Síntesis de las principales orientaciones de la formación docente propuesto por Navio (2006).

\begin{tabular}{|l|l|}
\hline Orientaciones & Características básicas \\
\hline $\begin{array}{l}\text { Orientación } \\
\text { académica }\end{array}$ & $\begin{array}{l}\text { Considera al docente como un especialista del contenido que debe transmitirse } \\
\text { en el proceso de enseñanza-aprendizaje. Así, la formación docente se basa } \\
\text { en el contenido con el fin de que se disponga de un dominio altamente } \\
\text { especializado de la materia. }\end{array}$ \\
\hline $\begin{array}{l}\text { Orientación } \\
\text { técnica }\end{array}$ & $\begin{array}{l}\text { El centro de interés en la formación docente está en el conocimiento del } \\
\text { contenido y en las destrezas (competencias) necesarias para la enseñanza. }\end{array}$ \\
\hline $\begin{array}{l}\text { Orientación } \\
\text { personalista }\end{array}$ & $\begin{array}{l}\text { Considera que el docente es un facilitador del aprendizaje del discente y, por } \\
\text { tanto, debe conocer las características básicas de éste. }\end{array}$ \\
\hline $\begin{array}{l}\text { Orientación } \\
\text { práctica }\end{array}$ & $\begin{array}{l}\text { Considera la enseñanza como una actividad compleja y contextualizada. Por } \\
\text { ello, el docente debe ser un profesional reflexivo que pueda dar respuestas } \\
\text { adaptadas a las situaciones complejas. El elemento fundamental de esta } \\
\text { orientación está en la reflexión que, desde la práctica, permite al profesional } \\
\text { intervenir y dar respuesta a los retos que se le plantean. }\end{array}$ \\
\hline $\begin{array}{l}\text { Orientación } \\
\text { social- } \\
\text { reconstruccionista }\end{array}$ & $\begin{array}{l}\text { A la reflexión se añade el espíritu crítico que debe tener el docente. De } \\
\text { hecho, la complejidad de proceso de enseñanza-aprendizaje requiere en el } \\
\text { profesional de la educación y de la formación no sólo de procesos de reflexión, } \\
\text { sino también de una actitud crítica frente a dicho proceso. Además, como } \\
\text { orientación ubicada en el paradigma ecológico, hace del contexto un elemento } \\
\text { básico sobre el que se asienta la reflexión y la crítica. Queremos hacer notar, } \\
\text { además, que la consideración del contexto es amplia. Por una parte, como } \\
\text { otras orientaciones apuntaban, el contexto es el de enseñanza-aprendizaje. } \\
\text { Pero también considera el contexto social en el que se desarrolla este proceso } \\
\text { sobre el que el profesional deberá actuar de manera crítica con una actitud } \\
\text { transformadora. }\end{array}$ \\
\hline
\end{tabular}

Tradicionalmente, una forma de aproximarnos al cómo aprenden a enseñar los formadores universitarios tiene relación con la noción de profesionalizar esta docencia, la cual se sustenta en el paradigma de que la mejora en la enseñanza ocurre necesariamente por el desarrollo profesional del docente universitario entendido como la mejora en su cualificación pedagógica (Imbernon, 1999; Manso, 1999; McLean y Blackwell, 1997).

Desde esta perspectiva se concibe que para poder enseñar es necesario fortalecer y desarrollar un conjunto de contenidos, habilidades, técnicas y estrategias pedagógicas, las cuales le permitirían llevar a cabo dicha tarea con mayor éxito, dado que la capacitación necesariamente mejora la enseñanza universitaria (Manso, 1999). 
Con respecto a la propuesta de Navio (2006), este tipo de orientación correspondería a los primeros tres descritos en la tabla 1 , en los cuales subyace una racionalidad de tipo técnica orientada al conocimiento experto (Schön, 1998). Esta perspectiva se puede asociar con el eje "Desarrollo de Personal Académico y Gestión Docente" de los Proyectos de Mejoramiento de la Calidad en Educación Superior "MECESUP 2: Educación Superior para la Sociedad del Conocimiento", el cual tiene como propósito fortalecer y profesionalizar a los docentes universitarios por medio de la creación y fortalecimiento de centros de desarrollo docente que se observan al interior de algunas universidades en nuestro país (MECESUP, 2010).

No obstante ello, si bien este tipo de iniciativas contribuyen a dimensionar las habilidades que debiesen poseer los formadores universitarios y son un espacio óptimo para la investigación de sus prácticas pedagógicas, al mismo tiempo pueden minimizar la complejidad de los procesos básicos de enseñanza y aprendizaje, ya que al conceptualizar la enseñanza como un repertorio específico de conductas o un enfoque genérico, la reduce a una "cuestión técnica de desempeño" sin tomar en cuenta su naturaleza relacional e interactiva (Healey y Jenkins, citado en Ashwin, 2006).

Por lo expuesto, se considera fundamental superar la imagen común y masificada de la docencia universitaria como una tarea sencilla, restringida a un conjunto de habilidades cognitivas fácilmente transferibles. Por el contrario, es necesario comenzar a conceptualizarla de manera más crítica, entendiendo así que el formador, además del dominio y conocimiento de una disciplina específica, reflexiona y sistematiza de manera intencionada un saber práctico respecto de cómo enseñar acorde a las nuevas demandas, tomando en consideración la complejidad del entorno así como también las características propias de los futuros profesionales que educan (Kreber, 2002b; Trigwell, 2001; Trigwell y Shale, 2004).

Desde esta nueva perspectiva, que se relaciona con los dos últimos tipos de orientaciones para la formación propuestos por Navio (2006), es importante destacar que se sustenta en una epistemología de la práctica desarrollada por un "profesional reflexivo", que se 
caracteriza por ser un investigador de su propia práctica profesional, que realiza un proceso continuo de autoaprendizaje, opuesto al concepto de "profesional experto", entendido como aquel que posee un rol preeminente producto de su autoridad y pericia en un conocimiento específico (Schön, 1995, 1998).

En nuestro país, esta perspectiva ha sido escasamente desarrollada y discutida ya que este modelo implica concebir un nuevo tipo de docente, el "formador reflexivo", que fortalece su capacidad de enseñar a futuros profesionales a través de la investigación y sistematización de su propia práctica como formador (Schön, 1992). Esto implica mirar la docencia de manera distinta, pues los profesionales adquieren la habilidad de enseñar por medio de una indagación realizada por ellos mismos más que por la transferencia o el entrenamiento realizado por otros.

No obstante, en países anglosajones como Estados Unidos, Inglaterra y Australia esta nueva perspectiva ya es un tema ampliamente discutido e investigado gracias al concepto "the scholarship of teaching", término acunado por Boyer (1990), que, como ha sido poco usado en países de habla hispana, no tiene una traducción consensuada al interior de nuestra comunidad científica.

Este concepto da cuenta de un tipo de conocimiento o saber específico relacionado con la forma de enseñar que es propia y particular de un área disciplinar y que se desarrolla por medio de una práctica reflexiva que es sistemática en el formador universitario (Kreber, 2002a, 2002b; Montenegro, 2009; Shulman, 2000; Trigwell y Shale, 2004). Dadas esas características, para efectos de este artículo se propone traducir el concepto como un "saber académico sobre la enseñanza", aludiendo a un tipo de conocimiento específico que desarrolla el formador respecto de la disciplina que enseña a través de un estudio intencionado.

Lo relevante de este concepto es que su discusión ha permitido fortalecer los mecanismos para la transmisión y enseñanza de conocimientos en la formación de los futuros profesionales por medio de una práctica reflexiva e investigativa, efectuada por los mismos formadores universitarios, orientada a comprender cómo los 
estudiantes aprenden la disciplina que se les enseña y de qué manera se pueden desarrollar prácticas pedagógicas que permitan mejores resultados de aprendizajes en ellos.

A su vez, este concepto invita a los formadores universitarios a generar una especie de didáctica de la propia disciplina que enseñan, dirigida no solo a transmitir conocimientos, sino también el ethos profesional que los define como profesión y que es compartida a la comunidad académica.

Al respecto, Kreber (2002b) plantea que los docentes universitarios que desarrollan un saber académico sobre la enseñanza en su práctica educativa se caracterizan por realizar una rendición pública de la totalidad de los aspectos que ésta contempla, de modo tal que dicha rendición permite por un lado la revisión por parte de miembros de la comunidad académica de este tipo de saber y, por otra, la posibilidad de que pueda ser utilizado o replicado por otros miembros de dicha comunidad. Esto implica:

"la construcción de un conocimiento acerca de la enseñanza a través de recursos formales y personales; combinan dicho conocimiento de manera efectiva con el conocimiento disciplinar que dominan para construir un conocimiento pedagógico del contenido que enseñan; desarrollan y fortalecen este tipo de conocimiento a través de un proceso de aprendizaje autorregulado; y validan este conocimiento desarrollado a través de la revisión de pares" (Kreber, 2002b, p. 18).

Por lo tanto, en la medida que los formadores universitarios desarrollen de manera sistemática este tipo de saber académico y lo compartan al interior de las comunidades educativas se observarían cambios no sólo en la forma en que se llevan a cabo los procesos de enseñanza y aprendizaje al interior de las salas de clases, sino que también en la forma que se concibe y define el rol de los formadores universitarios.

Esto implica modificar la imagen de los docentes universitarios como agentes que trasmiten y reproducen contenidos para comenzar a contemplarlos como profesionales reflexivos que, al investigar su 
práctica como formadores, desarrollan un tipo de conocimiento acerca de la compleja tarea de enseñar, tan fundamental como el desarrollo y producción de conocimientos de la disciplina que enseñan e investigan.

\section{Reflexiones finales: perspectivas y desafíos pendientes en este tema}

Profundizar en la tarea de formar a futuros profesionales es un tema que requiere levantar evidencias desde distintos niveles, uno de carácter relacional/formativo y otro asociado como insumo para la toma de decisiones en el ámbito de políticas de formación.

En el primer nivel es posible relevar la importancia de la relación que se establece entre el formador, sus habilidades para enseñar, y su vínculo con el estudiante; focalizando en los diversos tipo de prácticas que generan mejores oportunidades de aprendizaje en los futuros profesionales, así como también permiten la creación de un cuerpo común de conocimiento entre los formadores (Kreber, 2002a; Shulman, 1999; Yair, 2008; Boyer, 1990).

En este contexto, es posible abordar el tema desde los procesos reflexivos de base que permitan responder: cómo se enseña una disciplina profesional; de qué manera a través de la formación logramos transmitir su ethos a los futuros profesionales y qué habilidades requieren los formadores universitarios para lograrlo. Esto demanda una forma distinta de concebir los programas de formación, afectando tanto los procesos de enseñanza como los de aprendizaje en las instituciones universitarias (Shulman, 1999).

Lo anterior implica el paso a un segundo nivel, referido a la toma de decisiones, donde la constitución de comunidades reflexivas de formadores universitarios (Fuentealba y Galaz, 2008; Montenegro, 2009), se orienta a debatir, investigar y reflexionar las bases de la enseñanza más acordes a su disciplina, tomando en cuenta el núcleo de la profesión como un eje articulador que organiza las prácticas formativas; situación que, por lo demás, implica la generación de condiciones institucionales para el desarrollo de estas iniciativas. 
Los antecedentes expuestos, así como la evidencia empírica internacional ponen de manifiesto que un nuevo rumbo se debe comenzar a transitar en este tema, y que en Chile, si bien inicialmente se está comenzado a perfilar, falta mucho camino por recorrer. Dado eso, se considera fundamental que tanto los formadores como las personas que investigamos en educación superior nos interioricemos de estas temáticas y que aportemos al debate de la calidad de los aprendizajes, profundizando de qué manera nuestro país se preocupa y ocupa del tipo de docencia que se imparte al interior de la sala de clase; qué políticas públicas debiesen comenzar a perfilarse con relación al papel de los formadores universitarios, y de qué manera se incentivan y apoyan investigaciones relacionadas con los conocimientos y habilidades necesarios para enseñar en la formación profesional.

\section{Referencias bibliográficas}

Ashwin, Paul (2006) Changing Higher Education. The development of Learning $\&$ Teaching. London and New York: Routledge Taylor \& Francis Group.

Barnett, Ronald (2000) University knowledge in an age of supercomplexity. Higher Education, 40(4), pp. 409-422.

Becher, Tony (2001) Tribus y territorios académicos: la indagación intelectual y las culturas de las disciplinas. Barcelona: Gedisa.

Biggs, John (2003) Teaching for quality learning at university: what the student does. Philadelphia, Pa.: Society for Research into Higher Education: Open University Press.

Boyer, Ernest (1990) Scholarship Reconsidered: Priorities of the Professoriate. Princeton, NJ: Carnegie Foundation for the Advancement of Teaching.

Brew, A. (2003) Teaching and Research: New relationships and their implications for inquiry-based teaching and learning in Higher Education. Higher Education Research \& Development, 22(1), pp. 3-18.

Brunner, José Joaquín (2008) El proceso de Bolonia en el horizonte latinoamericano: límites y posibilidades. Revista de Educación Superior, pp. 119-145.

CNA (2010) Comisión Nacional de Acreditación. Consultado el 25 de mayo de 2010 desde http://www.cna.cl 
D'Andrea, Vaneeta y Gosling, David (2005) Improving Teaching and Learning in Higher Education. A whole institution approach. Great Britain: Society for Research into Higher Education \& Open University Press.

Fuentealba, Rodrigo y Galaz, Aberto (2008) La reflexión como recurso para la mejora de las prácticas docentes en servicio: el caso de las redes pedagógicas locales en Chile. En J. Cornejo \& R. Fuentealba (Eds.), Prácticas reflexivas para la formación profesional docente: ¿Qué las hace eficaces? Santiago de Chile: Ediciones UCSH.

Harland, T. y Staniforth, D. (2008) A family of strangers: the fragmented nature of academic development. Teaching in Higher Education, 13(6), pp. 669-678.

Imbernon, Francisco (1999) Responsabilidad social, profesionalidad y formación inicial en la docencia universitaria. Revista Interuniversitaria de Formación del Profesorado, 34, pp. 123-132.

Imbernon, Francisco (2000) Un nuevo profesorado para una nueva Universidad: ¿Conciencia o Presión? Revista Interuniversitaria de Formación del Profesorado, 38, pp. 37-46.

Knight, Peter (2005) El profesorado de Educación Superior: formación para la excelencia. Madrid: Narcea Ediciones.

Kreber, Carolin (2002a) Controversy and Consensus on the Scholarship of Teaching. Studies in Higher Education, 27(2), pp. 151-167.

Kreber, Carolin (2002b) Teaching Excellence, Teaching Expertise, and the Scholarship of Teaching. Innovative Higher Education, 27(1), pp. $5-23$.

Manso, José (1999) Profesionalización pedagógica del profesorado universitario. Revista Interuniversitaria de Formación del Profesorado, 34, pp. 319-328.

Martin, Elaine; Prosser, Michael; Trigwell, Keith et al. (2000) What university teachers teach and how they teach it. Instructional Science, 28, pp. 387-412.

McLean, Monica y Blackwell, Richard (1997) Opportunity Knocks? Professionalism and excellence in university teaching. Teachers and Teaching, 3(1), pp. 85-99.

MECESUP (2010) Programa de Mejoramiento de la Calidad y Equidad en Educación Superior. Consultado el 17 de mayo de 2010 desde http:// www.mecesup.cl/info_mece2/sitio/index.php

Montenegro, Helena (En prensa) Docencia en contextos de Educación Superior: la contribución de "the scholarship of teaching" para el fortalecimiento 
de la enseñanza llevada a cabo por profesores universitarios. Foro Educacional.

Navio, Antonio (2006) La formación de los profesionales de la formación para el trabajo: algunos dilemas y algunas respuestas. Revista Educar, 38, pp. 63-79.

OCDE (2009) Revisión de Políticas Nacionales de Educación: La Educación Superior en Chile. Chile: Organización para el Desarrollo y la Cooperación Económicas y el BIRD/Banco Mundial.

Prosser, Michael y Trigwell, Keith (1999) Understanding learning and teaching: the experience in higher education. Buckingham [England]: Society for Research into Higher Education \& Open University Press.

Ramsden, Paul (2003) Learning to Teach in Higher Education. London and New York: Routledge Falmer Taylor \& Francis Group.

Schön, Donald (1992) La formación de profesionales reflexivos. Hacia un nuevo diseño de la enseñanza y el aprendizaje de las profesiones. Barcelona: Paidós.

Schön, Donald (1995) The new scholarship requires a new epistemology. Change, 27(6), pp. 26-34.

Schön, Donald (1998) El profesional reflexivo: cómo piensan los profesionales cuando actúan. Barcelona: Paidós.

Shulman, Lee (1987) Knowledge and teaching. Foundations of the new reform. Harvard Education Review, 57(1), pp. 1-22.

Shulman, Lee (1999) Taking Learning Seriously. Change, 31(4), pp. 10-17.

Shulman, Lee (2000) From Minsk to Pinsk: Why A Scholarship of Teaching and Learning? Journal of the Scholarship of Teaching and Learning, 1(1), pp. $48-43$.

Trigwell, Keith (2001) Judging university teaching. The International Journal for Academic Development, 6(1), pp. 65-73.

Trigwell, Keith y Shale, Suzanne (2004) Student learning and the scholarship of university teaching. Studies in Higher Education, 29(4), pp. 523-536.

Yair, Gad (2008) Can we administer the scholarship of teaching? Lessons from outstanding professors in higher education. Higher Education, 55, pp. 447-459.

Recibido: 14/04/2010

Aceptado: 25/05/2010 